\title{
Editorial
}

\section{Classical and Hybrid Optimization Approaches and Their Applications in Engineering and Economics}

\author{
Pandian Vasant, ${ }^{1}$ Gerhard-Wilhelm Weber, ${ }^{2}$ and Vo Ngoc Dieu ${ }^{3}$ \\ ${ }^{1}$ Department of Fundamental and Applied Sciences, Universiti Teknologi PETRONAS, 32610 Bandar Seri Iskandar, Malaysia \\ ${ }^{2}$ Institute of Applied Mathematics, METU, 06531 Ankara, Turkey \\ ${ }^{3}$ Department of Power Systems, HCMC University of Technology, Ho Chi Minh City, Vietnam
}

Correspondence should be addressed to Pandian Vasant; pvasant@gmail.com

Received 9 April 2015; Accepted 9 April 2015

Copyright (c) 2015 Pandian Vasant et al. This is an open access article distributed under the Creative Commons Attribution License, which permits unrestricted use, distribution, and reproduction in any medium, provided the original work is properly cited.

Classical optimization and, for some years, hybrid optimization have been successfully applied to many problems of engineering and economics. For instance, as exposed and detailed in the literature, gravitational search algorithm (GSA), genetic algorithm (GA), particle swarm optimization (PSO), ant colony optimization (ACO), and several hybrid swarm evolutionary algorithms could be adopted to overcome complex and uncertain real-world optimization challenges. What is more, advances in hybrid optimization techniques are an important section in engineering and economics and they also assist optimization algorithm experts to design and code better procedures. In order to bridge the notions, concepts, and methodologies between those two ends, our special issue focussed on the related topics of integrating and utilizing algorithms in hybrid computational intelligent techniques and their applications in engineering and economics. The hybrid systems can be a hybrid between the classical methods and artificial intelligence-based methods. This special issue may provide the opportunity for practitioners to handle their complicated real-world problems by using hybrid optimization methodologies and for researchers to realize their significant contributions, conveying their knowledge and insights into practice, to look and propose future directions.

This special issue aimed at providing a forum for adopting the state-of-the-art hybrid optimization techniques in engineering and economics, developing the advanced hybrid optimization techniques by using metaheuristics approaches, exchanging of related ideas, and discussing the future directions. A special attention was assigned to exchange, comparison, and combination of the classical and more mathematical and model-based methods of optimization with the many emerging model-free methods from computer science. By this, we aimed to strengthen the mathematical and engineering sciences and to contribute to markets, industries, and economies and, eventually, to the living conditions in the world. We welcomed researchers to present their newest original works, and so many experts, both scholars and practitioners, from all over the world followed our invitation.

Topics of this special issue included, but also went much beyond, the following:

(i) classical optimization methods of mathematics and their applications in engineering and economics;

(ii) continuous optimization applications in engineering and economics;

(iii) combinatorial optimization applications in engineering and economics;

(iv) mixed-integer programming applications in engineering and economics;

(v) hybrid optimization with metaheuristics techniques multiobjective hybrid optimization approaches handling uncertainties with hybrid optimization;

(vi) Lagrange optimization;

(vii) Kuhn-Tucker optimization;

(viii) chaotic hybrid optimization;

(ix) linear and nonlinear optimization;

(x) mathematical programming; 
(xi) theoretical aspects of hybrid optimization methods;

(xii) emerging real-world and theoretical applications in engineering and economics.

\section{Acknowledgments}

The guest editors are very grateful to the editorial team and entire staff, for their confidence, interest, continuous guidance, and support at all levels of preparation of this special issue's preparation. Furthermore, we would like to sincerely thank Universiti Teknologi PETRONAS, METU, and HCMC University of Technology for their marvellous support and encouragement. We, guest editors, wish all the readers a pleasant and enjoyable, insightful and inspiring lecture of the contributions of this special issue. In fact, we cordially hope that this special issue will present and value this journal as a premium journal in science, engineering, economics, and finance, which strongly fosters deeply needed intellectual advances and their contributions to humankind in all over the world.

Pandian Vasant Gerhard-Wilhelm Weber Vo Ngoc Dieu 


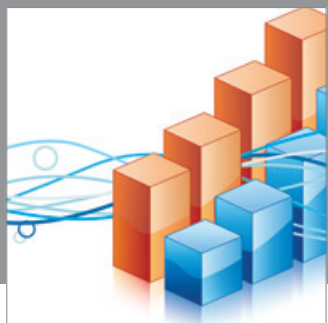

Advances in

Operations Research

mansans

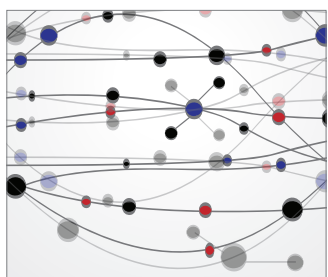

The Scientific World Journal
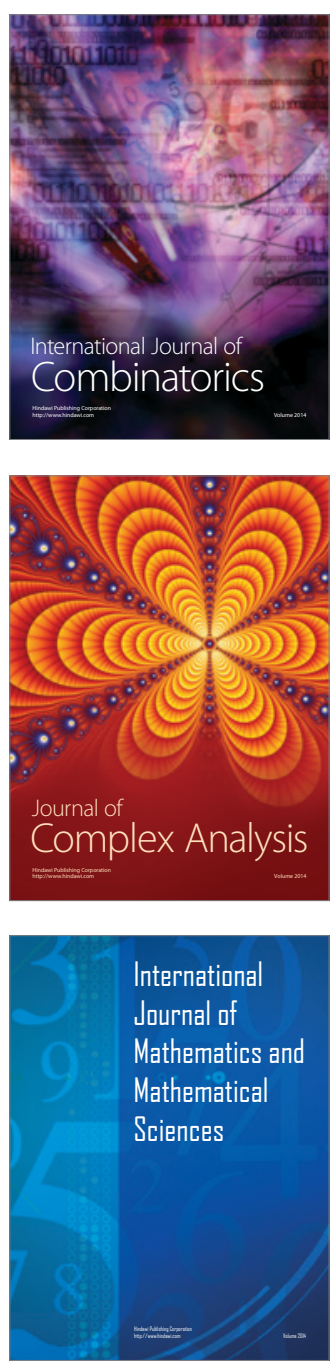
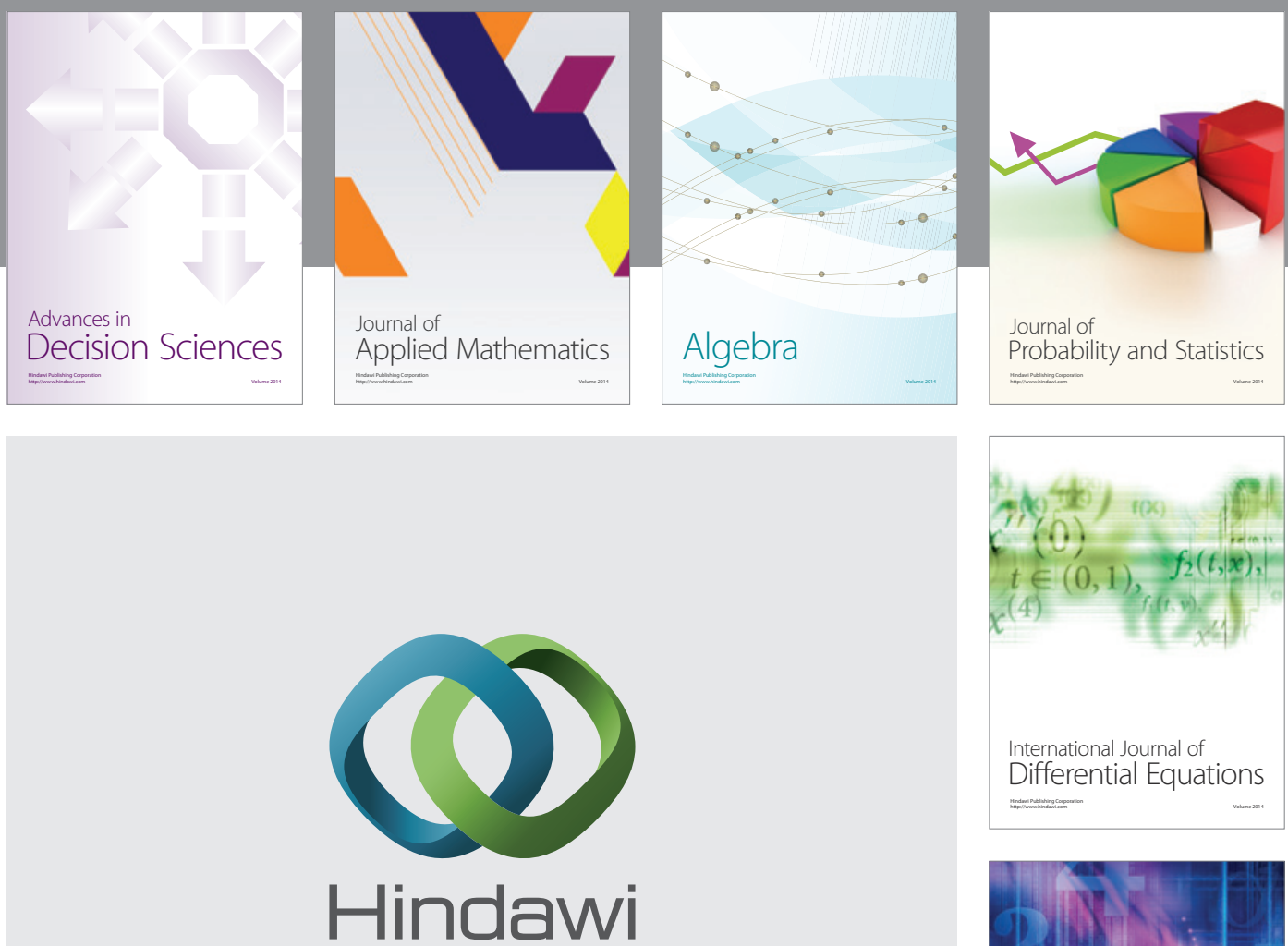

Submit your manuscripts at http://www.hindawi.com
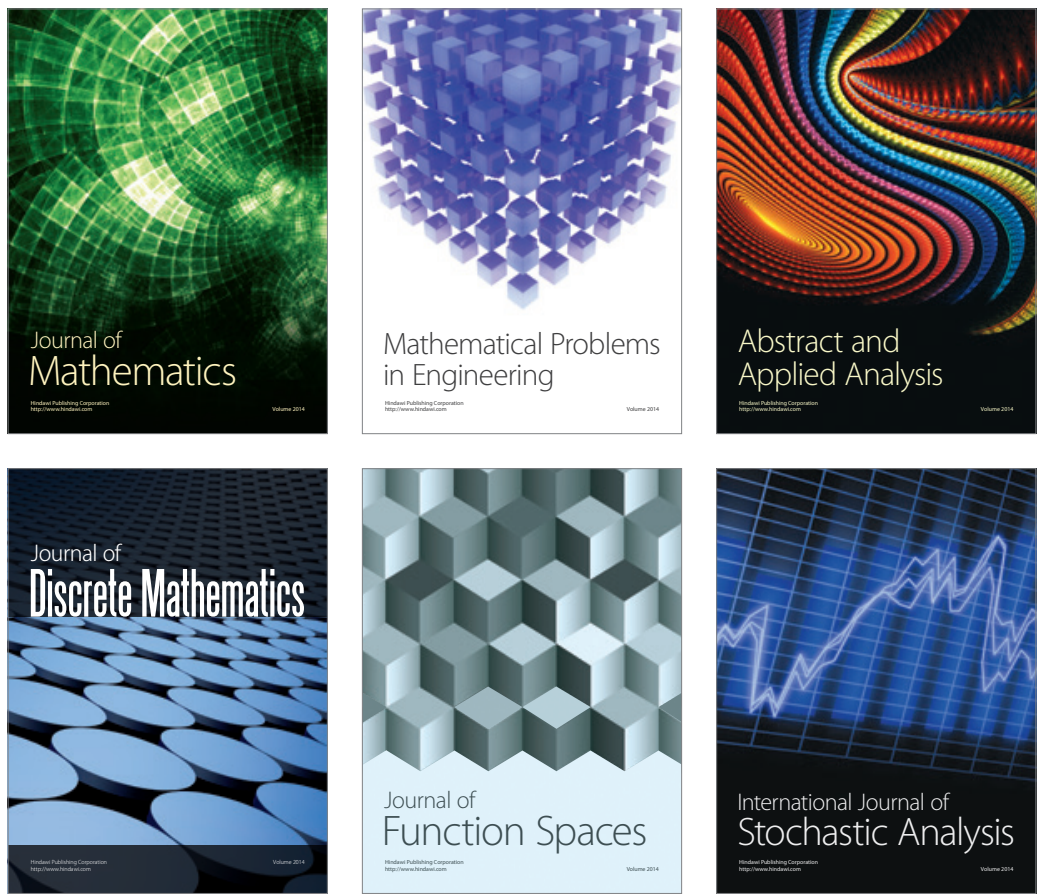

Journal of

Function Spaces

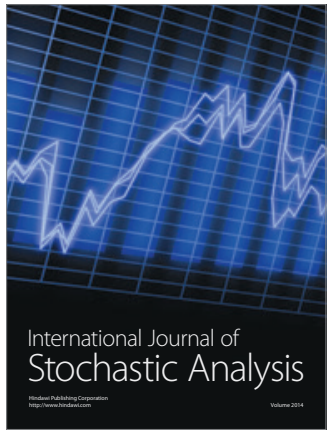

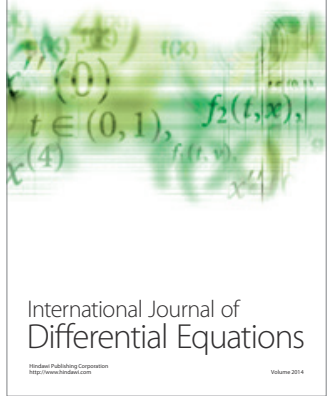
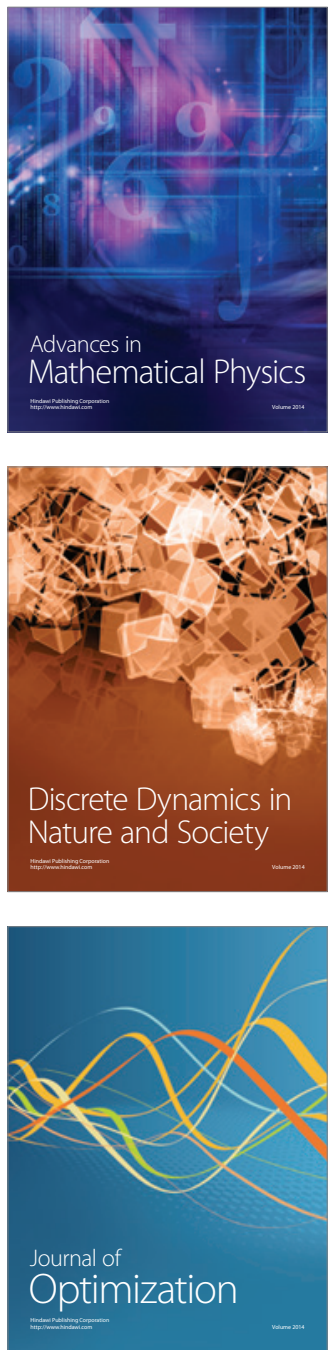